\title{
The Role of the Guanine-Based Purinergic System in Seizures and Epilepsy
}

\author{
André P. Schmidt and Diogo O. Souza*
}

\author{
Department of Biochemistry, Instituto de Ciências Básicas da Saúde (ICBS), Universidade Federal do Rio Grande do \\ Sul, Porto Alegre, RS, Brazil
}

\begin{abstract}
Guanine-based purines have been traditionally studied as modulators of intracellular processes, mainly Gprotein activity. However, more recently, several studies have shown that they exert a variety of extracellular effects not related to G-proteins, including trophic effects on neural cells, modulation of glutamatergic activity, behavioral effects and anticonvulsant activity. In this article, the putative effects of the guanine-based purines against seizures and neurotoxicity are reviewed. Current evidence suggests that guanine-based purines, especially guanosine, seem to be endogenous anticonvulsant substances, perhaps in a similar way to the adenine-based purines. Although studies addressing the mechanism of action of guanine-based purines are still lacking, their anticonvulsant activity is probably related to the modulation of several glutamatergic parameters, especially the astrocytic glutamate uptake. These findings point to the guaninebased purines as potential new targets for the development of novel drugs for neuroprotection and management of epilepsy.
\end{abstract}

Keywords: Guanine-based purines, seizures, epilepsy, adenine-based purines, guanosine, purines, neuroprotection, glutamate.

\section{INTRODUCTION}

The purinergic system usually relates to the adeninebased purines, including the nucleotides adenosine 5'triphosphate (ATP), adenosine 5'-diphosphate (ADP) and adenosine 5'-monophoshate (AMP), and the nucleoside adenosine. Adenine-based purines exert several biological roles, including the pivotal role on energy metabolism. Extracellular adenosine and ATP are usually considered the major endogenous effectors of the purinergic system, acting on $\mathrm{P} 1$ and $\mathrm{P} 2$ receptors, respectively [1]. However, guaninebased purines, namely the nucleotides guanosine 5'triphosphate (GTP), guanosine 5'-diphosphate (GDP) and guanosine 5'-monophosphate (GMP) and the nucleoside guanosine also are a relevant component of the purinergic system.

Traditionally, guanine-based purines have been studied as intracellular modulators of signal transduction processes, modulating the activity of G-proteins [2]. Nevertheless, more recently, guanine-based purines have been shown to exert relevant extracellular effects, including those related to the modulation of the glutamatergic system [3-12]. Although the exact mechanism underlying these extracellular effects remains unclear, it does not seem to involve a direct modulation of G-proteins [12]. Guanine-based purines were shown to inhibit the binding of glutamate and analogs $[3,6,7,13]$, to be neuroprotective under excitotoxic conditions [14-16], as well as anticonvulsant against seizures induced by glutamatergic agents [10,11,17-20]. Notably, the effects of guaninebased purines on animal models of seizures were one of the

*Address correspondence to this author at the Avenida Ramiro Barcelos, 2600-Anexo, Zip code 90035-003, Porto Alegre - RS - Brazil; Tel: (55-51) 33085558; Fax: (55-51) 33085540/33085535; E-mail: diogo@ufrgs.br first direct and reliable evidences of an in vivo modulation of the glutamatergic system by those substances $[6,11]$.

In this article, the putative effects of the guanine-based purines in seizures and neurotoxicity are briefly reviewed, with emphasis on their potential role in neuroprotection and epilepsy management.

\section{GUANINE-BASED PURINERGIC SYSTEM}

\subsection{Historical Overview}

In 1971, the first evidence for a more complex class of signaling pathway emerged establishing that the sensor and intracellular effector are separate proteins that communicate through proteins called guanine nucleotide-dependent regulatory proteins, GTP binding proteins or G-proteins [21]. Gproteins alternate between active GTP-bound and inactive GDP-bound forms. Activation is catalyzed by receptors and deactivation by an intrinsic property of G-proteins, its GTPase activity. G-proteins couple cell surface receptors to cellular effectors, modulating cell responses to external stimuli: the interaction of agonists with their receptors triggers the binding of GTP to G-proteins, forming an active complex G-protein/GTP, which simultaneously modulates the activity of effector systems and decreases the agonist binding to specific receptors [2].

Recently, it has become increasingly recognized that guanine-based purines have also important extracellular signalling effects, including in vitro inhibitory effects on the activity of the glutamatergic system, trophic effects on neural cells, neuroprotection against ischemic insults, effects on learning and memory, modulation of pain pathways, anticonvulsant effects and other behavioral alterations [reviewed in ref. 12]. Before reviewing the putative aspects of guaninebased purines on seizures and epilepsy animal models, we 
will summarize some aspects related to the synthesis and metabolism of the main constituents of the guanine-based purinergic system.

\subsection{Synthesis and Metabolism of Guanine-Based Purines}

Extracellular purine nucleosides and nucleotides are ubiquitous intercellular messengers, which may affect several biological functions, largely acting as neurotransmitters or neuromodulators $[1,16]$. Several lines of evidence indicate that guanine-based purines, mainly the nucleotide GTP and the nucleoside guanosine, act as intercellular signalling molecules, as well as their counterparts ATP and adenosine $[16,22]$. In the nervous system guanine-based purines mediate both immediate effects, such as neurotransmission, and trophic effects which induce changes in cell metabolism, structure and function.

Adenine- and guanine-based purines share some metabolic steps (i.e., nucleoside transporters and ectonucleotidases). Therefore, it is tempting to propose that guanine- and adenine-based purines may respond similarly in certain conditions. Astrocytes are the main sources of extracellular adenine- and guanine-based purines in the central nervous system (CNS) [22]. They are largely involved in several brain functions in physiological conditions, participating in neuronal development, synaptic activity, homeostatic control of the extracellular environment and also in processes related to brain injuries, by arresting and repairing further neural damage [23,24]. Additionally, growing evidence indicates that purines are widely involved in the molecular mechanisms underlying the multiple functions of astrocytes, either exerting their influence on key intracellular activities (energy metabolism and nucleic acid synthesis) or activating a variety of membrane receptors [25]. Cultured astrocytes release guanine-based purines, a process that is importantly augmented after hypoxic and low glucose insults [22]. Interestingly, the release of guanine-based purines seems to be larger than that observed for adenine-based purines [22]. Actually, guanine-based purines are released in amounts about 3 -fold greater that their adenine-based counterparts and the amounts of guanine-based purines, especially guanosine, is further augmented when the cells are exposed to a brief period of hypoxia/hypoglycemia [22]. Notably, purine nucleosides and nucleotides are extensively released from degenerating cells, particularly under hypoxic and ischemic conditions. This latter is particularly relevant in the CNS, where purines may depress neurotransmission, thus reducing excitotoxic neuronal damages [26], and may regulate the responses of nervous tissue to injury.

The presence of adenine- and guanine-based purines and their metabolites in human and animal cerebrospinal fluid (CSF) has been well described [27-30]. Astrocytes, as well as neurons, are responsible for both nucleoside metabolism and uptake of the nucleosides adenosine and guanosine [31]. Following their release, essentially from astrocytes, the extracellular levels of the various purine nucleotides and nucleosides are regulated by the activities of cell surfacelocated enzymes, which have the same function of the corresponding intracellular enzymes [32]. The enzymes involved in extracellular nucleotide hydrolysis include membranebound ecto-nucleotidases, ecto-nucleotidases released from membranes and the naturally occurring soluble nucleoti- dases. These enzymes, in association with ecto-5'nucleotidase, hydrolyze extracellular nucleotides in a stepwise fashion down to nucleosides and are crucial for physiological modulation of CNS functions, as well as for the purine-induced neuroprotection [33]. Several such enzymes (ectonucleotidases) comprise the ecto-nucleoside triphosphatase (E-NTPase) family. E-NTPases include the ecto-ATPase that preferentially converts ATP into ADP; the ecto-ATP diphosphohydrolase (ectoapyrase) that hydrolyses either ATP or ADP, and the ecto-5'-nucleotidase that catalyses the hydrolysis of AMP to adenosine [34,35]. Nevertheless, the selectivity for adenine-based purines is not complete, as these enzymes also hydrolyse all purine and pyrimidine nucleotides, including guanine-based purines. In regard to the nucleosides, adenosine is deaminated by adenosine deaminase (ADA) and guanosine is converted to guanine by guanase. These soluble nucleotidases are also present and active in rat CSF [36,37], where they hydrolyze all guanine and adenine nucleotides with the following order of catalytic efficiency: GDP $>$ ADP $=$ ATP = GTP $>$ AMP = GMP [30]. Interestingly, at high concentrations, GDP hydrolysis rate is greater than that of ADP, perhaps favoring the accumulation of GMP and guanosine. In fact, these enzymes can be released to the extracellular space (CSF) from choroid plexus, endothelial cells or even microglia and play an important regulatory role of the purinergic system under physiological and pathological conditions [34,35]. Notably, in cultured astrocytes, inhibition of ecto-5'-nucleotidase activity significantly impaired accumulation of extracellular guanosine, indicating that, similar to extracellular adenosine, it is to some extent derived from the extracellular metabolism of guanine nucleotides [38].

Nucleosides are also removed from the extracellular space into neurons and glia by transporter systems. Uptake of purine and pyrimidine nucleosides by astrocytes is also important for nucleic acid synthesis and synthesis of AMP, ADP, and ATP from adenosine and GTP from guanosine [39]. Peng et al. [40] has identified two equilibrative nucleoside transporters in astrocytes $\left(\mathrm{ENT}_{1}\right.$ and $\left.\mathrm{ENT}_{2}\right)$, together with the concentrative nucleoside transporter $\left(\mathrm{CNT}_{2}\right)$ responsible for nucleoside uptake. Interestingly, an equilibrative nucleoside transporter $\left(\mathrm{ENT}_{1}\right)$ was also recently identified in the rat brain endothelial cells and choroid plexus epithelial cells, indicating a more ubiquitous distribution of the purine nucleoside transmembrane transport system [41].

Growing evidence suggests that guanine-based purines interact at the level of signal-transduction pathways with other transmitters, for example, glutamate and GABA [42]. It is commonly accepted that purines production in the brain is closely related to the release of neurotransmitters and that $\mathrm{K}^{+}$-induced depolarization evokes the release of endogenous neurotransmitters which, in turn, promote purine outflow [43,44]. Released nucleotides and nucleosides are considered to act as retrograde synaptic transmitters, modulating the release of several putative neurotransmitters, including glutamate and GABA. However, to date, little is known about the potential influence of guanine-based purines on neuronal function and synaptic plasticity and new studies are warranted.

Regarding the CNS guanosine bioavailability, a single intracerebroventricular (i.c.v.) administration of GMP in mice 
causes a large increase in the CSF levels of GMP, guanosine and oxypurines (hypoxanthine, xanthine and uric acid) [45]. We also observed that intraperitoneal (i.p.) administration of GMP in anticonvulsant doses produced a 3-fold increase of CSF levels of guanosine in rats, not affecting GMP levels [46]. Intracerebroventricular administration of guanosine did not affect GMP and hypoxanthine CSF levels, in spite of causing a significant increase in guanosine, xanthine and uric acid CSF levels [45]. Intrathecal (i.t.) administration of guanosine produced a significant increase in guanosine, inosine, xanthine and uric acid CSF levels [47]. Notably, the significant increase of CSF concentration of oxypurines after i.c.v. or i.t. injection of guanosine probably indicates an in vivo degradation. Additionally, animals treated with oral guanosine in anticonvulsant doses presented a 2-fold increase in CSF concentration of guanosine as compared to control [19]. Intraperitoneal or oral administration of highdose guanosine (up to $120 \mathrm{mg} \cdot \mathrm{kg}^{-1}$ ) produced a 6.8 or 7.8 fold increase in guanosine CSF levels, respectively [48]. Importantly, guanosine CSF levels remained increased up to 360 min after a single i.p. administration of guanosine in rats [49]. However, systemic guanosine did not affect the CSF levels of inosine, oxypurines and adenine-based purines [48]. Notably, a previous study has demonstrated that an i.p. administration of guanosine increased the amounts of both guanosine and guanine in the spinal cord, with a peak around $30 \mathrm{~min}$ [50]. Considering that extracellular guanine also exerts several biological effects [51,52], it is tempting to propose that some biological effects of guanosine may be regulated by its conversion to guanine by a membrane located purine nucleoside phosphorylase.

\subsection{Effects of Guanine-Based Purines Against Seizures and Toxicity in Animals}

Glutamate is the major excitatory neurotransmitter in the mammalian CNS, participating in plastic processes involved in learning and memory [53], development and aging [54] and environmental adaptation [55]. However, glutamate may also be a potent neurotoxin and overstimulation of the glutamatergic system (by exogenous or endogenous stimuli), which occurs when extracellular glutamate levels increase over the physiological range, has been implicated in the pathogenesis of various acute and chronic CNS disorders [56-58]. Consequently, the equilibrium between the physiological and pathological glutamatergic tonus is essential for brain function and its disruption is related with the pathogenesis of various CNS disorders including the epilepsies [56,59-61].

It is now clearly shown that glutamatergic excitotoxicity is prevented by astrocytic glutamate uptake, a process responsible for maintaining the extracellular glutamate levels below toxic levels $[23,58,61]$. Since adenosine decreases glutamate release and guanosine increases glutamate uptake (and persists for longer periods of time extracellularly), both purine nucleosides may act in concert to reduce the impact of glutamate-induced excitability. This issue might be especially important in the endogenous and exogenous modulation of glutamate-related seizures. Glutamate undoubtedly plays a pivotal role on epilepsy and probably in other CNS disorders precipitating seizure activity [56-58,62,63]. However, the cellular and molecular mechanisms involved in the generation and maintenance of seizures and toxicity are not fully understood.

Guanine nucleotides, intracerebroventricularly administered, had long been shown to prevent seizures induced by quinolinic acid, a toxin that overstimulates the glutamatergic neurotransmission [6]. This effect was compatible with the antagonistic properties of guanine nucleotides on glutamate receptors being studied in our group [3]. However, after further exploring the interaction of guanine nucleotides with glutamate, we observed that besides the administration of
A

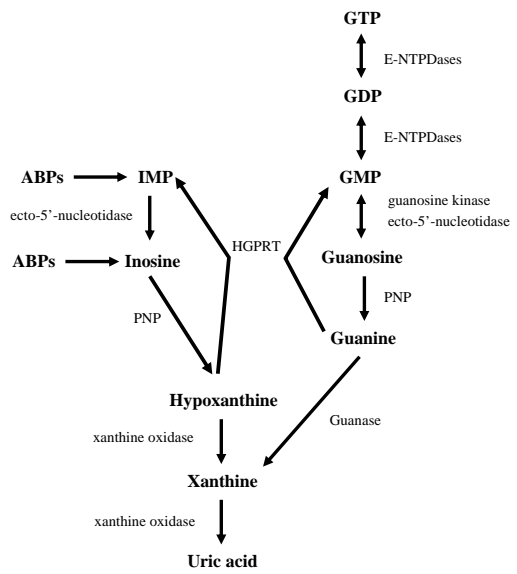

B

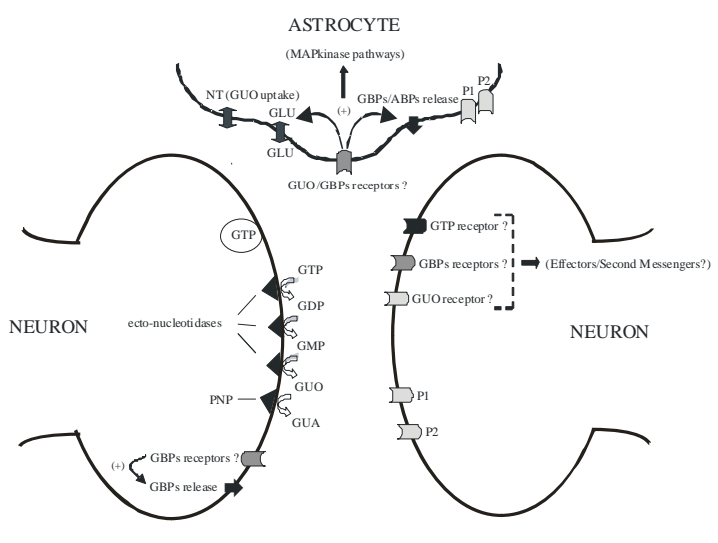

Fig. (1). Panel A: Schematic model of the sources and different enzymes pathways of extracellular guanine-based purines. ABPs $=$ adeninebased purines; E-NTPDases = ecto-nucleotide-diphosphohydrolase; PNP = purine nucleoside phosphorylase; HGPRT = hypoxanthineguanine phosphoribosyltransferase. Panel B: Schematic representation of the guanine-based purinergic system. Once released from astrocytes, guanine-based purines may interact with its specific receptors on neurons and astrocytes, exerting trophic effects and modulating neurotransmitters release and uptake. "?" is referred to the potential existence of specific receptors for guanine-based purines; MAPkinase = Mitogen-activated protein kinase; NT = nucleoside transporters; GUO = guanosine; GUA = guanine; GLU = glutamate; GBPs = guaninebased purines. 
GMP, guanosine also prevented seizures induced by quinolinic acid [11]. Later we showed that a single i.c.v. administration of GTP and GDP was also protective against seizures induced by quinolinic acid in mice [18]. Notably, we also observed that single and chronic oral administrations of guanosine were also effective in the same seizure model [10]. Importantly, quinolinic acid is a direct NMDA agonist, but also stimulates glutamate release and inhibits its uptake [64]. Additional studies also provided evidence that guanosine and GMP administered intracerebroventricularly, intraperitonially or orally dose-dependently protected against seizures induced by the other glutamatergic agents such as kainic acid and $\alpha$-dendrotoxin in adult and young rodents $[17,19,20,46,65,66]$. The fact that chronic oral exposure to guanine-based purines produced anticonvulsant effects indicates that these substances are orally active in the long term and points to a future target for new antiepileptic drugs in adults and children. Of note, chronically administered GMP had also been shown to be neuroprotective against quinolinic acid-induced striatal neuronal cell death in rats [15], strengthening the notion that the guanine-based purinergic system may be a valuable target for the treatment of neurodegenerative disorders. Table 1 summarizes the main studies addressing the effects of guanine-based purines on seizures and toxicity induced by glutamate and its analogs in vitro and in vivo.

Guanine-based purines, mainly GMP and guanosine, have usually presented similar neuroprotective profile in several in vivo and in vitro protocols [10,11,18-20,64]. However, most effects of nucleotides (mainly GMP) seemed to be due to their conversion to guanosine. Specifically for seizures, an acute i.c.v. administration of the ecto-5'nucleotidase (enzyme that converts GMP to guanosine) inhibitor AOPCP prevented the anticonvulsant effects of GMP against quinolinic acid in rats, without affecting the effect of guanosine [46]. Moreover, we demonstrated that anticonvulsant effects of i.c.v. GTP and GDP seemed to be mediated by their conversion to guanosine, since their poorly hydrolysable analogs GTP $\gamma$ S, GppNHp and GDP $\beta S$ were not capable of preventing seizures induced by quinolinic acid in mice [18]. Of note, a recent study demonstrated that GMP-induced antinociception was also prevented by AOPCP, corroborating with anticonvulsant effects [45].

Although the behavioral effects of guanosine in preventing quinolinic acid-induced seizures have been well described $[10,11,17,18]$, little is known about its electrophysiological effects in the brain. Recently, we performed the first study addressing this issue based on epidural electroencephalogram (EEG) recordings of rats [67]. In addition to clear EEG changes occurring during the seizure events, we found that quinolinic acid disrupted a prominent basal theta (4-10 $\mathrm{Hz}$ ) activity during peri-ictal periods and promoted a relative increase in the power level at the gamma band; guanosine, when successfully preventing seizures, counteracted this effect following quinolinic acid administration. Interestingly, we observed that MK-801, a known NMDA-antagonist used as a positive control, presented different spectral effects when compared to guanosine in rats protected against quinolinic acid-induced seizures, producing large gamma oscillations following quinolinic acid administration [67]. Additionally, the combined pre-treatment with both guanosine and MK-801 in this model led to qualitatively different re- sults than the observed when each drug was administered alone [67]. Considering these recent evidences, we suggest that a diverse mechanism of action between both drugs (guanosine versus MK-801 or perhaps other NMDA antagonists) exists and that guanosine might be related to a lower incidence of cognitive side effects than NMDA antagonists in the clinical setting.

It is well known that guanine-based purines, mainly guanosine, are protective against quinolinic acid-induced seizures in a dose-dependent manner $[11,18]$. However, it still remains puzzling why these substances are not effective, even in high doses, in preventing quinolinic acid-induced seizures in $100 \%$ of the cases. In previous studies, we have found that the most effective doses of guanine-based purines accounts for nearly $50 \%$ of protection in the quinolinic acidinduced seizures model $[10,11,18,67]$. Importantly, Torres et al. [67] observed that this somewhat partial anticonvulsant effect does not seem to be related to individual differences among animals, suggesting that dynamic variables within rats are probably determining whether guanine-based purines will successfully prevent seizures or not. Importantly, this study has demonstrated that protected and non-protected animals under guanosine treatment can be distinguished electrophysiologically, even before the beginning of the motor seizures. Accordingly, we found that the level of theta power greatly decreased in animals displaying seizures under guanosine treatment, both before and after the seizure event, similarly to what we observed in vehicle pretreated rats. On the other hand, animals successfully protected by guanosine exhibited a higher level of theta power than animals displaying seizures. It is possible that these variables are related to pharmacokinetic and pharmacodynamic factors, although it could also be related to the current internal state of the brain.

Besides epilepsy and general seizure behavior, brain ischemia is responsible for significant morbidity and mortality and significant resources have also been dedicated to developing new neuroprotective strategies. Since adeninebased purines have been demonstrated to play a role in endogenous neurodegenerative and neuroprotective processes [68], guanine-based purines could also be investigated for possible therapeutic manipulations. As stated above (section 2.2), using primary cultures of astrocytes prepared from the rodent cerebral hemispheres, it was shown that they spontaneously release guanine-based purines even in basal conditions. Interestingly, the amount of guanine-based purines (especially guanosine) released over a 3-hour period was greater than that of adenine-based purines [22]. Moreover, the exposure of these cultures to hypoxia/low glucose levels resulted in sustained increase in the release of guanine- and adenine-based purines over basal values up to 90 minutes after the insult. Notably, the release of purines was not related to an artifact of diminished cell viability [22]. These effects in an in vitro ischemia/stroke model are consistent with the hypothesis that these compounds may exert pivotal modulatory effects on synaptic transmission and more sustained trophic effects.

Guanine-based purines have also recently been shown to play a role in the Lesch-Nyhan syndrome [69] and perhaps in several other neurodegenerative diseases [70,71]. The rationale for this hypothesis is based on clinical and biochemical characterization of some neurological disorders such as 
Table 1. Summary of Main Experimental Studies Investigating the Guanine-Based Purines on Seizures and Glutamatergic Toxicity In Vitro Studies

Inhibit kainic acid binding

Souza and Ramirez, 1991[3]; Paz et al., 1994[7]; Ramos et al., 1997[9]

Prevent cell responses to excitatory amino acids Burgos et al., 1998[4]; 2000[5]

Inhibit glutamate and its analogs binding

Rubin et al., 1997[52]; Baron et al., 1989[6]

Prevent NMDA-induced excitotoxicity

Baron et al., 1989[6]; Molz et al., 2008[100]; Caciagli et al., $2000[38]$

Stimulate glutamate uptake by astrocytes

Frizzo et al., 2001[107]; 2003[102]; Gottfried et al., 2002[112]

Stimulate the glutamate uptake by synaptic vesicles

Tasca et al., 2004[8]

Stimulate glutamate uptake in brain slices

Frizzo et al., 2002[14]; 2005[110]; Thomazi et al., 2004[113]

Prevent the decrease of glutamate uptake induced by hypoxic-ischemic insult

Moretto et al., 2005[114]

Prevent quinolinic acid-induced release of glutamate on synaptosomes

Prevent neural apoptosis

Preserve neural viability in mouse spinal cord cultures during chemical hypoxia

Tavares et al., 2005[65]

Di Iorio et al., 2004[70]; Pettifer et al., 2004[71]

Litsky et al., 1999[123]

Protect glial cells against glucose deprivation and mitochondrial inhibition

Jurkowitz et al., 1998[124]

Reduce apoptosis and stimulates neurogenesis in rats with parkinsonism

Su et al., 2009[52]

Reduce apoptosis and inflammation in rats with acute spinal cord injury

Jiang et al., 2007[134]

\section{In Vivo Studies}

Prevent quinolinic acid-induced seizures in mice

Prevent quinolinic acid-induced seizures in young rats

Prevent quinolinic acid-induced seizures in adult rats

Prevent quinolinic acid-induced neural death

Prevent seizures induced by several glutamatergic agents in rodents

Electrophysiological effects of guanosine against quinolinic acid-induced seizures in rats

Induce transitory amnesia in rats

Induce transitory amnesia in mice

Prevent the facilitatory effect of glutamate on memory in rats

Chronic treatment is anxiolytic in mice

Attenuate hyperlocomotion induced by MK-801

Prevent MK-801-induced hyperalgesia

Prevent in vivo decrease of astrocytic glutamate uptake induced by quinolinic acid

Improve locomotor function and remyelination in rats submitted to a spinal cord injury model

Produce antinociceptive effects against glutamatergic pain models in rodents Improve motor behavior in rats with parkinsonism

Neuroprotective effects against stroke

Schmidt et al., 2000[11]; 2005[18]; Lara et al., 2001[10]

de Oliveira et al., 2004[17]

Soares et al., 2004[46]

Malcon et al., 1997[15]

Lara et al., 2001[10]; Vinadé et al., 2003[20]

Torres et al., 2010[67]

Roesler et al., 2000[77]; Saute et al., 2006[79]

Vinadé et al., 2003[10]; Vinadé et al., 2004[78]

Rubin et al., 1996[76]

Vinadé et al., 2003[20]

Tort et al., 2004[80]

Schmidt et al., 2009[83]

Vinadé et al., 2005[19]

Jiang et al., 2003[135]; 2007[134]

Schmidt et al., 2008[45]; 2009[47,49]; 2010[48]

Su et al., 2009[52]

Chang et al., 2008[136]

Lesch-Nyhan syndrome and the neurobiological consequences of the hypoxanthine phosphoribosyltransferase (HPRT) deficiency. Conceivably, diminished reutilization of free guanine bases due to absent or reduced HPRT activity and relatively high guanase activity in the brain could lead to deficient endogenous pools of guanosine associated with 
glutamatergic synapses. These issues demonstrated the potential roles that guanine-based purines play in neurodevelopment and as neuromodulators. Nevertheless, these findings remain to be further investigated but strongly suggest guanine-based purines as potential drug targets in the experimental therapy of neuroinflammatory and neurodegenerative diseases.

Considering that purines, their metabolites and the soluble nucleotidases responsible for their hydrolysis are detected in the human and animal CSF and blood serum $[27,29,36,72,73]$, and their potential role on an "endogenous neuroprotection system", it is possible that these parameters may be new putative markers of CNS injury. We have demonstrated that pentylenetetrazol-induced seizures promote an increase in CSF nucleotidases activity represented by further hydrolysis of GDP and ADP and an increase in concentration of guanosine and inosine (probably related to quick degradation of adenosine to inosine) 30 minutes after the insult [74]. Increases of GDP/ADP hydrolysis and levels of nucleosides guanosine/inosine after pentylenetetrazol-induced seizures presented a somewhat similar profile to other wellknown brain injury markers (S100 $\beta$-protein and neuronspecific enolase - NSE). This temporal similarity suggests that those compounds could become biochemical brain markers to evaluate neural injury.

\subsection{Behavioral Effects of Guanine-Based Purines}

It has been well demonstrated for several glutamate antagonists, mainly NMDA-receptor antagonists, that they may induce amnesia and severe locomotor deficits in animals [75]. It is well documented that glutamate plays a key role on memory mechanisms [53], and previous studies demonstrated that GMP was able to reverse the facilitatory effect of post-training intra-hippocampal glutamate administration on inhibitory avoidance task performance in rats [76]. Further studies demonstrated that GMP and guanosine are capable to modulate memory processes since pretraining administration of both guanine-based purines impaired retention of inhibitory avoidance responses in rats [20,77]. The guanine-based purine effects on memory were reproduced with anticonvulsant doses after acute/chronic i.p./oral administration and adenosine-receptor antagonists failed to prevent these effects [78]. Furthermore, the amnesic effect related to the pretreatment with GMP also depended on its conversion to guanosine [79]. These findings suggest an amnesic effect of guanosine on inhibitory avoidance in rodents, in a pattern compatible with inhibition of glutamatergic activity and independent of adenosine $A_{1}$ and $A_{2 A}$ receptors.

Most studies have indicated that guanosine per se does not affect spontaneous locomotion in rodents [9,20,80]. Additionally, no obvious motor disturbance or sedative effects were observed since acute or chronic administration of guanine-based purines did not alter rotarod and open field performance, as evidenced with other glutamate antagonists such as MK-801 [9,20]. Interestingly, NMDA-receptor antagonists have been related to significant locomotor disturbances [81]. Recent evidence suggest that NMDA receptor antagonism may be associated with glutamatergic activation of non-NMDA glutamatergic receptors induced by increased glutamate release, which appears to be closely related to those behavioral alterations [82]. Therefore, despite of reduc- ing glutamatergic effects at NMDA receptors, NMDAreceptor antagonists may stimulate non-NMDA receptors by increasing the release of glutamate. Interestingly, guanosine produced an approximately $60 \%$ attenuation of hyperlocomotion induced by MK-801 (a non-competitive NMDAreceptor antagonist), whereas it did not affect the hyperlocomotion induced by the indirect dopamine agonist amphetamine or by the non-selective adenosine-receptor antagonist caffeine [80]. Additionally, we have recently observed that MK-801 induces paradoxical hyperalgesia in the rat tail-flick paradigm, a behavior effect significantly correlated with increased CSF levels of the excitatory amino acids glutamate and aspartate [83]. Notably, guanosine prevented both neurochemical and behavioral effects induced by MK801 [83]. The attenuation of some behavioral effects of MK801 by guanosine (locomotor and nociceptive effects) may be related to an increase of glutamate uptake by astrocytes promoted by guanosine, reducing glutamate levels at the synaptic cleft and leading to less activation of non-NMDA receptors $[83,84]$.

More recently, our group has demonstrated that guaninebased purines produce consistent antinociceptive effects against several pain models, including those based on thermal or chemical stimuli $[45,47-49,84,85]$. These antinociceptive effects were investigated by using the nucleotide GMP and the nucleoside guanosine, but other guanine-based purines might cause such effects as well. Interestingly, we also demonstrated that GMP-induced antinociception was prevented by the ecto-5'-nucleotidase inhibitor $\alpha, \beta$ methyleneadenosine 5'-diphosphate (AOPCP), suggesting that its effects result from conversion to guanosine [45]. Our studies clearly demonstrated that i.c.v., i.t. or systemically (i.p.) administered guanosine produces significant inhibition of pain-related behavior induced by several algogens in mice [45,47-49,84,85]. Additionally, i.t. or i.p. guanosine prevents biting behavior induced by i.t. administration of glutamate and non-NMDA agonists, but it was not effective against NMDA $[47,48]$. We also demonstrate that these antinociceptive effects may involve some adenosine receptors $\left(\mathrm{A}_{1}\right.$ and $\mathrm{A}_{2 \mathrm{~A}}$ ) and spinal cord glutamate uptake [48].

The contribution of adenosine $A_{1}$ and $A_{2 A}$ receptors to the effects of guanosine has also been ruled out in some behavioral studies. The adenosine antagonist caffeine failed to inhibit the anticonvulsant effect of an acute orally administration of guanosine on quinolinic acid-induced seizures in mice [10] or the amnesic effect of guanosine in rats $[77,78]$. Conversely, more recently, we demonstrated that a pretreatment with non-selective (Caffeine) and selective $A_{1} / A_{2 A}$ receptor antagonists (DPCPX and SCH58261) significantly affected guanosine-induced nociception [48]. Allopurinolinduced antinociception, an event related to the accumulation of guanosine and adenosine in the CSF, was prevented by caffeine and DPCPX as well [85]. Therefore, at least for antinociception, adenosine receptors seem to be relevant to guanine-based purine effects, but further work is warranted.

\subsection{Insights into the Mechanism of Action of Guanine- Based Purines}

It has been classically demonstrated that by acting via Gproteins, GTP is able to simultaneously inhibit binding of neurotransmitters (and their agonists) to metabotropic recep- 
tors and modulate adenylate cyclase activity $[2,86]$. However, we have demonstrated that the effects of guanine nucleotides on kainic acid binding site and on adenylate cyclase activity could be dissociated [3]. In lysed membrane preparations, the guanine nucleotides GMP, GDP and GTP were able to inhibit the binding of kainic acid with the same efficiency, whereas only GTP was able to stimulate cell membrane adenylate cyclase activity. However, in vesicular preparations, all guanine nucleotides were still able to inhibit binding of kainic acid, whereas GTP lost the ability to stimulate adenylate cyclase activity. These findings strongly suggested that the inhibition of kainic acid binding by guanine nucleotides was not dependent on a G-protein-mediated system. This result corroborated studies from other groups, which had previously shown that the inhibitory effects of guanine nucleotides on the binding of glutamate or ionotropic glutamatergic ligands presented several inconsistencies, when compared with studies on receptors known to be coupled to their second messengers through a G- protein [6,87-90]. Subsequent studies from our group supported the hypothesis that guanine nucleotides could antagonize the glutamatergic transmission by acting at extracellular sites located on the membrane surface [91-94].

Searching for a relevance of the inhibitory action of extracellular guanine nucleotides on glutamate binding, several studies further investigated their putative effects on neural cell responses to glutamate and/or analogs [7,95-101]. It was observed that guanine nucleotides inhibited glutamatestimulated GFAP (glial fibrillary acidic protein) phosphorylation [96], glutamate (and analogs)-induced modulation of intracellular cAMP levels [97,98], kainate-stimulated lactate dehydrogenase (LDH) release [4], kainate-activated currents $[94,101]$ and kainate-stimulated increase in $\mathrm{Ca}^{2+}$ influx [93]. Since most excitatory synapses in the CNS have glutamate as neurotransmitter, the potential modulatory action of guanine nucleotides on the glutamatergic neurotransmission claimed attention to new investigations on their extracellular roles.

Several studies have indicated that guanine-based purines, especially guanosine, may be neuroprotective endogenous compounds released under excitotoxic conditions, preventing further toxicity to neural cells [reviewed in ref. 12]. Considering that guanine-based purines seem to be especially effective after conversion to guanosine $[45,65,79,102]$ and the fact that this nucleoside probably exerts only weak glutamate receptor antagonism, the hypothesis of direct receptor interaction as the mechanism of neuroprotection and anticonvulsant action of guanine-based purines is unlikely, although this issue deserves further investigation $[103,104]$.

Guanosine occurs naturally in the brain and has been reported to present numerous biological effects when administered extracellularly, including trophic effects on neural cells (mainly astrocytes) [16,39] and modulation of glutamatergic activity [12]. Guanosine stimulates the release of adeninebased purines from astrocytes, which may be responsible for some effects of guanine-based purines [105]. For example, the ability of guanine-based purines to stimulate proliferation of rat brain microglia in a concentration-dependent manner appears to be mediated by specific purinergic receptors that recognize adenine-based purines [105]. But this explanation is also incomplete, since many of the effects of guaninebased purines persist in the presence of $\mathrm{P}_{1}$ and/or $\mathrm{P}_{2}$ purine receptor antagonists $[45,99,106,107]$. An alternative hypothesis is that there are distinct receptors for guanine-based purines. Although those effects might be related to guanosine uptake into the intracellular compartment, a consensus has emerged that at least some effects of guanosine involve its binding to a specific membrane protein $[108,109]$, postulated to be a $\mathrm{G}$ protein-coupled receptor $[16,108,109]$. Moreover, several of the effects of guanosine may be mediated through G-protein dependent signalling pathways involving changes in the intracellular levels of cyclic nucleotides or mitogen-activated protein kinase (MAPK) pathway raising the possibility that some of the effects of guaninebased purines, particularly guanosine, involve activation of cell-surface receptors $[26,106]$. Indeed, the actual existence of its putative specific receptor has yet to be demonstrated. More certain is the fact that guanosine presents clear antiglutamatergic properties, as demonstrated in several in vivo and in vitro approaches [reviewed in ref. 12], which places it as a new potential neuroprotective strategy against glutamatergic excitotoxicity.

The mechanism of action underlying the modulation of glutamatergic activity by guanosine, and perhaps other guanine-based purines, is currently under research in our and other laboratories. It has been suggested that astrocytes are importantly involved, since guanosine has been shown to stimulate glutamate uptake by cultured astrocytes and brain slices $[14,102,107,110]$. Astrocytic glutamate uptake is a crucial process for the maintenance of extracellular glutamate concentrations below toxic levels in physiological conditions and under brain stress, thus supporting synapse homeostasis (glutamate-glutamine cycle) [23]. Actually, this is the main mechanism of glutamate removal from the synaptic cleft [61]. Notably, both neuronal and astrocytic cell cultures are able to release guanosine under basal or ischemic conditions [16,22,111] and kainate stimulates the release of guanosine [111]. In physiological conditions, the effects of guanosine on glutamate uptake in brain slices seem to be age (more in young animals) and structure (more in cortex) dependent but, in excitotoxic conditions, guanosine seems to be more widely involved in modulating glutamate uptake $[14,110,112,113]$. In cultured primary astrocytes from cortices of 1-day-old and adult rat brain cortical slices, guanosine was shown to increase the sodium-dependent uptake of glutamate in a dose-dependent manner [107]. Importantly, adenosine affected neither the basal uptake nor the stimulatory effect of guanosine. Theophylline, a nonspecific $\mathrm{P}_{1}$ $\left(\mathrm{A}_{1} / \mathrm{A}_{2 \mathrm{~A}}\right)$ adenosine-receptor antagonist, stimulated basal uptake of glutamate without affecting the stimulatory effect of guanosine. Finally, dipyridamole, a nucleoside transport inhibitor, also stimulated basal glutamate uptake, and this stimulatory effect was additive with that of guanosine. Thus, these findings suggest that the guanosine stimulatory effect on astrocytic uptake of glutamate is exerted from the extracellular side and is, at least partially, independent of the adenosinergic system [107].

GMP and GTP mimicked the stimulatory effect of guanosine on glutamate uptake by astrocytic cells in culture [102]. However, a significant additive effect on uptake was not observed with the simultaneous addition of guanosine, GMP and GTP to the culture medium, compared with the 
effect of each compound alone. These data were consistent with the possibility that only one compound was mediating the stimulatory effect on uptake or the three compounds were metabolically interconvertible with each other. Importantly, a poorly hydrolysable analogue of GTP did not stimulate the uptake of glutamate by cultured astrocytes and the effect of GMP was abolished when cultures were pretreated with AOPCP. Finally, guanosine failed to affect the astrocytic uptake of GABA [102]. Therefore, guanosine seems to be mediator of the stimulatory effect of guanine-based purines on the astrocytic uptake of glutamate, and this process was independent of adenosine and relatively specific for glutamate [102]. As astrocytic uptake of glutamate is the most important mechanism for terminating its actions within the synapse, the stimulation of uptake by guanosine may be a relevant process in regulating glutamatergic neurotransmission, especially under excitotoxic conditions [23,58,61].

Oral administration of guanosine prevented the decrease of glutamate uptake by brain slices of rats submitted to quinolinic acid seizure model [17,20]. Additionally, guanosine has been shown to prevent the decrease of glutamate uptake by hippocampal slices of neonatal rats exposed to a hypoxicischemic insult in vivo [114]. Moreover, we demonstrated that in vitro and in vivo quinolinic acid stimulated synaptosomal glutamate release and inhibited glutamate uptake by astrocytes, which could lead to increased extracellular glutamate levels and seizures [64,115]. However, this neurochemical effect was prevented by in vivo pretreatment with systemic guanosine or GMP [65]. Additionally, quinolinic acid stimulates glutamate uptake by synaptic vesicles, an effect prevented by glutamate antagonists and the guaninebased purines guanosine and GMP [66]. Altogether these findings indicate that glutamate uptake modulation induced by guanosine might play a crucial role in the underlying mechanisms involved in the anticonvulsant effects of guanine-based purines.

In addition to their effects on neurotransmission, guanine-based purines also have important trophic functions, affecting the development, structure or maintenance of neural cells [39]. Of note, guanosine has been shown to stimulate the release of neurotrophic factors, an event largely related to cell proliferation [16]. Some trophic effects of purines seem to be mediated via purinergic cell surface receptors, whereas others require uptake of purines by the target cells [39]. Guanosine and GTP, apparently through different mechanisms, are related to several trophic events, including stimulation of astroblast growth [116], in vitro axonal growth and proliferation of a wide range of cell types $[117,118]$, and trophic effects on the CNS, including stimulation of astrocyte proliferation, induction of synaptogenesis [119], synthesis and release of trophic factors such as nerve growth factor from astrocyte cultures, and differentiation of PC12 cells and hippocampal neurons in vitro $[38,39,105,106,116,120,121]$. Some of the trophic actions of guanine-based purines may be indirect, occurring as a result of stimulating the synthesis and release of trophic factors and/or enhancing the effects of these specific trophic factors. Another possibility is that some actions of guanosine could be mediated intracellularly after its uptake. However, with respect to a specific neurotrophic role for guanosine, its extracellular levels remained elevated for up to a week after focal brain injury [122]. Additionally, many trophic effects of guanine-based purines were not affected by the nucleoside uptake inhibitors, such as dipyridamole, indicating that they are triggered extracellularly [120].

The potential ability of exogenously administered guanine-based purines to provide an alternative source of energy to ATP has been suggested as an explanatory hypothesis for their neuroprotective effects in the context of oxidative stress and cell damage $[16,123]$. For example, after exposure to rotenone, an inhibitor of the mitochondrial respiratory chain, and the induction of chemical hypoxia, guanosine was shown to preserve the viability of cultured astrocytes and neurons $[123,124]$. The ability of guanosine to maintain cellular levels of ATP above a critical threshold under hypoxia may provide an explanation of the mechanism of their cell damage prevention. Indeed, the addition of a purine nucleoside phosphorylase inhibitor to the cultures, which would interfere with a pathway for the participation of purine nucleosides in the production of ATP under anaerobic conditions, attenuated their protective effect; this effect of purine nucleosides to preserve cell viability was especially dramatic with neurons. The data also suggested that neuronal protection by purine nucleosides is either dependent on or enhanced by the presence of glia [123].

\section{CONCLUSIONS AND PERSPECTIVES}

This article reviews the evidences about the potential anticonvulsant activity displayed by some components of the guanine-based purinergic system, perhaps providing new targets for neuroprotection and epilepsy treatment in children and adults. Guanine-based purines seem to modify the homeostasis of the glutamatergic system, modulating some glutamatergic parameters such as glutamate uptake by astrocytes and seizures induced by glutamatergic agents. Furthermore, the profile of extracellular activity of the guaninebased purines (endogenous compounds, orally active and no obvious CNS side effects except for transitory memory impairment) makes this system a very interesting object for discovery of new pharmacological options to treat diseases related to overstimulation of glutamatergic system such as epilepsy and other neurodegenerative diseases.

More specifically, to advance in guanine-based purine research, further studies are necessary on their mechanisms of action, cloning of putative selective receptors and characterization of second messengers related to their extracellular effects. In fact, the investigation of the extracellular effects of guanine-based purines and their underlying mechanisms of action is still in its infancy and advance is urgently warranted. This is especially true for the so called guanosine receptor characterization, which is pivotal for the proposal of a new neuromodulation pathway. Additional elucidation of the mechanism of action of guanosine and its membranebinding site is under current investigation in our laboratory.

Furthermore, little information about potential side effects and systemic toxicity of these compounds is available. A recent study showed a minor toxic potential of guanosine in mice, displaying an absent mortality index and lack of changes in weight body gain or core temperature up to $72 \mathrm{~h}$ after guanosine systemic administration, even in high doses [48]. However, this study has demonstrated some indication of liver toxicity (elevated liver enzymes) induced by guanosine in doses higher than $240 \mathrm{mg} \cdot \mathrm{kg}^{-1}$ [48]. Although 
these effects were not observed at antinociceptive doses, future studies may focus on potential adverse effects of guanosine including those involved on liver metabolism. In fact, specific studies about the safety profile of these compounds are pivotal for their future use in a clinical basis.

Although it is early to propose the use of guanine-based purines for clinical research, an interesting approach to investigate their role clinically is the investigation of purine derivatives already used in humans. For example, we have demonstrated that allopurinol, a xanthine oxidase inhibitor, was an effective and well-tolerated adjuvant treatment for poorly responsive schizophrenia, refractory aggressive behavior and mania [125-129]. These results were confirmed by an independent group $[130,131]$ and are hypothesized to be due to an indirect increase in extracellular purine levels (adenosine and guanosine) [12]. Notably, refractory epilepsy may also respond to allopurinol $[132,133]$. More recently, we have demonstrated that allopurinol produced dosedependent antinociceptive effects in several animal pain models [85]. The non-selective adenosine-receptor antagonist caffeine and the selective $\mathrm{A}_{1}$ adenosine-receptor antagonist, DPCPX, but not the selective $\mathrm{A}_{2 \mathrm{~A}}$ adenosine-receptor antagonist, SCH58261, completely prevented allopurinolinduced antinociception. Allopurinol also caused an increase in CSF levels of purines, including the nucleosides adenosine and guanosine, and decreased CSF levels of uric acid. Allopurinol-induced antinociception may be related to adenosine, and perhaps guanosine, accumulation. Considering that allopurinol is an old and extensively used compound and seems to be well tolerated with no obvious CNS toxic effects, allopurinol may be the first commercially available effective drug enhancing the effects of the purinergic systems for the treatment of human brain diseases, including chronic pain, psychiatric disorders and epilepsy. These findings indicate that new studies addressing more selective xanthine oxidase inhibitors in neuroprotection could represent a fine approach to investigate the therapeutic potential of purine in a clinical setting.

In conclusion, the role of the guanine-based purines as new targets for brain protection remains to be fully characterized, but current evidence strongly suggests their potential for the treatment of brain diseases such as epilepsy.

\section{ACKNOWLEDGEMENTS}

Supported by INCT for Excitotoxicity and Neuroprotection/CNPq, by the FINEP research grant Rede Instituto Brasileiro de Neurociências (IBN-Net) - 01.06.0842-00 and by CNPq, CAPES, FAPERGS and UFRGS.

\section{REFERENCES}

[1] Burnstock G. Purine and pyrimidine receptors. Cell Mol Life Sci 2007; 19: 1-13.

[2] Johnston CA, Siderovski DP. Receptor-mediated activation of heterotrimeric G-proteins: current structural insights. Mol Pharmacol 2007; 72: 219-30.

[3] Souza DO, Ramirez G. Effects of guanine nucleotides on kainic acid binding and on adenylate cyclase in chick optic tectum and cerebellum. J Mol Neurosci 1991; 3: 39-45.

[4] Burgos JS, Barat A, Souza DO, Ramírez G. Guanine nucleotides protect against kainate toxicity in an ex vivo chick retinal preparation. FEBS Lett 1998; 430: 176-80.

[5] Burgos JS, Barat A, Ramirez G. Guanine nucleotides block agonist-driven $45 \mathrm{Ca}^{2+}$ influx in chick embryo retinal explants. Neuroreport 2000; 11: 2303-5.
[6] Baron BM, Dudley MW, McCarty DR, Miller FP, Reynolds IJ, Schmidt CJ. Guanine nucleotides are competitive inhibitors of NMethyl-D-Aspartate at its receptor site both in vitro and in vivo. J Pharmacol Exp Ther 1989; 250: 162-9.

[7] Paz MM, Ramos M, Ramirez G, Souza D. Differential effects of guanine nucleotides on kainic acid binding and on adenylate cyclase activity in chick optic tectum. FEBS Lett 1994; 355: 205-8.

[8] Tasca CI, Santos TG, Tavares RG, Battastini AM, Rocha JB, Souza DO. Guanine derivatives modulate L-glutamate uptake into rat brain synaptic vesicles. Neurochem Int 2004; 44: 423-31.

[9] Ramos M, Souza DO, Ramirez G. Specific binding of $[3 \mathrm{H}] \mathrm{GppNHp}$ to extracellular membrane receptors in chick cerebellum: possible involvement of kainic acid receptors. FEBS Lett 1997; 406: 114-8.

[10] Lara DR, Schmidt AP, Frizzo ME, Burgos JS, Ramirez G, Souza DO. Effect of orally administered guanosine on seizures and death induced by glutamatergic agents. Brain Res 2001; 912: 176-80.

[11] Schmidt AP, Lara DR, Maraschin JF, Perla AS, Souza DO. Guanosine and GMP prevent seizures induced by quinolinic acid in mice. Brain Res 2000; 864: 40-3.

[12] Schmidt AP, Lara DR, Souza DO. Proposal of a guanine-based purinergic system in the mammalian central nervous system. Pharmacol Ther 2007; 116: 401-16.

[13] Paas Y, Devillers-Thiery A, Changeux JP, Medevielle F, Teichberg VI. Identification of an extracellular motif involved in the binding of guanine nucleotides by a glutamate receptor. EMBO J 1996; 15: $1548-56$.

[14] Frizzo ME, Lara DR, Prokopiuk AS, et al. Guanosine enhances glutamate uptake in brain cortical slices at normal and excitotoxic conditions. Cell Mol Neurobiol 2002 ; 22: 353-63.

[15] Malcon C, Achaval M, Komlos F, et al. GMP protects against quinolinic acid-induced loss of NADPH-diaphorase-positive cells in the rat striatum. Neurosci Lett 1997; 225: 145-8.

[16] Ciccarelli R, Ballerini P, Sabatino G, et al. Involvement of astrocytes in purine-mediated reparative processes in the brain. Int $\mathbf{J}$ Devl Neurosci 2001; 19: 395-414.

[17] de Oliveira DL, Horn JF, Rodrigues JM, et al. Quinolinic acid promotes seizures and decreases glutamate uptake in young rats: reversal by orally administered guanosine. Brain Res 2004; 1018: 48-54.

[18] Schmidt AP, Ávila TT, Souza DO. Intracerebroventricular guaninebased purines protect against seizures induced by quinolinic acid in mice. Neurochem Res 2005; 30: 69-73.

[19] Vinadé ER, Schmidt AP, Frizzo ME, et al. Effects of Chronic Administered Guanosine on Behavioral Parameters and Brain Glutamate Uptake in Rats. J Neurosci Res 2005; 79: 248-53.

[20] Vinadé ER, Schmidt AP, Frizzo ME, Izquierdo I, Elizabetsky E, Souza DO. Chronically administered guanosine is anticonvulsant, amnesic and anxiolytic in mice. Brain Res 2003; 977: 97-102.

[21] Rodbell M, Birnbaumer L, Pohl SL, Kraus HM. The glucagonsensitive adenyl cyclase system in plasma membranes of rat liver: An obligatory role of guanylnucleotides in glucagon action. J Biol Chem 1971; 246: 1877-82.

[22] Ciccarelli R, Di Iorio P, Giuliani P, et al. Rat cultured astrocytes release guanine-based purines in basal conditions and after hypoxia/hypoglycemia. Glia 1999; 25: 93-8.

[23] Chen Y, Swanson RA. Astrocytes and brain injury. J Cereb Blood Flow Metab 2003; 23: 137-49.

[24] Bélanger M, Magistretti PJ. The role of astroglia in neuroprotection. Dialogues Clin Neurosci 2009; 11: 281- 95.

[25] Abbracchio MP, Burnstock G. Purinergic signalling: pathophysiological roles. Jpn J Pharmacol 1998; 78: 113-45.

[26] Rudolphi KA, Schubert P, Parkinson FE, Fredholm BB. Neuroprotective role of adenosine in cerebral ischaemia. Trends Pharmacol Sci 1992; 13: 439-45.

[27] Castro-Gago M, Camina F, Lojo S, Rodriguez-Segade S, Rodriguez-Nunez A. Concentrations of purine nucleotides and purine and pyrimidine bases in cerebrospinal fluid of neurologically healthy children. Eur J Clin Chem Clin Biochem 1992; 30: 761-5.

[28] Kuracka L, Kalnovicová T, Líska B, Turcáni P. HPLC method for measurement of purine nucleotide degradation products in cerebrospinal fluid. Clin Chem 1996; 42:756-60.

[29] Regner A, Crestana RE, Silveira FJ, Friedman G, Chemale I, Souza D. Guanine nucleotides are present in human CSF. Neuroreport 1997; 8: 3771-4. 
[30] Rodríguez-Núñez A, Cid E, Rodríguez-García J, Camiña F, Rodríguez-Segade S, Castro-Gago M. Neuron-specific enolase, nucleotides, nucleosides, purine bases, oxypurines and uric acid concentrations in cerebrospinal fluid of children with meningitis. Brain Dev 2003; 25: 102-6.

[31] Parkinson FE, Xiong W, Zamzow CR. Astrocytes and neurons: different roles in regulating adenosine levels. Neurol Res 2005; 27: 153-60.

[32] Ceballos G, Tuttle JB, Rubio R. Differential distribution of purine metabolizing enzymes between glia and neurons. J Neurochem 1994; 62: 1144-53.

[33] Sebastião AM, Cunha RA, Cascalheira JF, Ribeiro JA. Adenine nucleotides as inhibitors of synaptic transmission: role of localized ectonucleotidases. Prog Brain Res 1999; 120: 183-92.

[34] Zimmermann H. Ectonucleotidases in the nervous system. Novartis Found Symp 2006; 276: 113-28.

[35] Zimmermann H. Nucleotide signaling in nervous system development. Pflugers Arch 2006; 452: 573-88.

[36] Cruz Portela LV, Oses JP, Silveira AL, et al. Guanine and adenine nucleotidase activities in rat cerebrospinal fluid. Brain Res 2002; 950: 74-8.

[37] Busnello JV, Oses JP, da Silva RS, et al. Peripheral nucleotide hydrolysis in rats submitted to a model of electroconvulsive therapy. Prog Neuropsychopharmacol Biol Psychiatry 2008; 32: 182933.

[38] Caciagli F, Di Iorio P, Giuliani P, Middlemiss MP, Rathbone MP. The neuroprotective activity of guanosine involves the production of trophic factors and the outflow of purines from astrocytes. Drug Develop Res 2000; 50: 32.

[39] Rathbone MP, Middlemiss PJ, Gysbergs JW, et al. Trophic effects of purines in neurons and glial cells. Prog Neurobiol 1999; 59: 66390.

[40] Peng L, Huang R, Yu AC, Fung KY, Rathbone MP, Hertz L. Nucleoside transporter expression and function in cultured mouse astrocytes. Glia 2005; 52: 25-35.

[41] Redzic ZB, Biringer J, Barnes K, et al. Polarized distribution of nucleoside transporters in rat brain endothelial and choroid plexus epithelial cells. J Neurochem 2005; 94: 1420-6.

[42] Di Iorio P, Ballerini P, Caciagli F, Ciccarelli R. Purinoceptormediated modulation of purine and neurotransmitter release from nervous tissue. Pharmacol Res 1998; 37: 169-78.

[43] Hoehn K, White TD. Role of excitatory amino acid receptors in K and glutamate-evoked release of endogenous adenosine from rat cortical slices. J Neurochem 1990; 54: 256-65.

[44] Craig CG, White TD. N-Methyl-D-Aspartate- and Non-N-MethylD-Aspartate-evoked adenosine release from rat cortical slices: distinct purinergic sources and mechanisms of release. J Neurochem 1993; 60: 1073-80.

[45] Schmidt AP, Böhmer AE, Leke R, et al. Antinociceptive effects of intracerebroventricular administration of guanine-based purines in mice: evidences for the mechanism of action. Brain Res 2008; 1234:50-8.

[46] Soares FA, Schmidt AP, Farina M, et al. Anticonvulsant effect of GMP depends on its conversion to guanosine. Brain Res 2004; 1005: 182-6.

[47] Schmidt AP, Böhmer AE, Schallenberger C, et al. Spinal mechanisms of antinociceptive action caused by guanosine in mice. Eur $\mathbf{J}$ Pharmacol 2009; 613: 46-53.

[48] Schmidt AP, Böhmer AE, Schallenberger C, et al. Mechanisms involved in the antinociception induced by systemic administration of guanosine in mice. Br J Pharmacol 2010; 159(6): 1247-63.

[49] Schmidt AP, Paniz L, Schallenberger C, et al. Guanosine prevents thermal hyperalgesia in a rat model of peripheral mononeuropathy. J Pain 2010; 11(2): 131-41.

[50] Jiang S, Fischione G, Guiliani P, Romano S, Caciagli F, Diiorio P. Metabolism and distribution of guanosine given intraperitoneally: implications for spinal cord injury. Nucleosides Nucleotides Nucleic Acids 2008; 27: 673-80.

[51] Rathbone M, Pilutti L, Caciagli F, Jiang S. Neurotrophic effects of extracellular guanosine. Nucleosides Nucleotides Nucleic Acids 2008; 27: 666-72.

[52] Su C, Elfeki N, Ballerini P, et al. Guanosine improves motor behavior, reduces apoptosis, and stimulates neurogenesis in rats with parkinsonism. J Neurosci Res 2009; 87: 617-25.

[53] Izquierdo I, Bevilaqua LR, Rossato JI, Bonini JS, Medina JH, Cammarota M. Different molecular cascades in different sites of

$496-505$.
[54] Segovia G, Porras A, Del Arco A, Mora F. Glutamatergic neurotransmission in aging: a critical perspective. Mech Ageing Dev 2001; 122: 1-29.

[55] Ozawa S, Kamiya H, Tsuzuki K. Glutamate receptors in the mammalian central nervous system. Prog Neurobiol 1998; 54: 581-618.

[56] Meldrum BS. The role of glutamate in epilepsy and other CNS disorders. Neurology 1994; 44: S14-23.

[57] Maragakis NJ, Rothstein JD. Glutamate transporters: animal models to neurologic disease. Neurobiol Dis 2004; 15: 461-73.

[58] Sheldon AL, Robinson MB. The role of glutamate transporters in neurodegenerative diseases and potential opportunities for intervention. Neurochem Int 2007; 51: 333-55.

[59] Bradford HF. Glutamate, GABA and epilepsy. Prog Neurobiol 1995; 47: 477-511.

[60] Chapman AG. Glutamate and epilepsy. J Nutr 2000; 130: 1043S5S.

[61] Beart PM, O'Shea RD. Transporters for L-glutamate: an update on their molecular pharmacology and pathological involvement. Br J Pharmacol 2007; 150: 5-17.

[62] Lipton SA, Rosemberg PA. Excitatory amino acids as a final common pathway for neurologic disorders. N Engl J Med 1994; 330: 613-22.

[63] Allen NJ, Karadottir R, Attwell D. Reversal or reduction of glutamate and GABA transport in CNS pathology and therapy. Pflugers Arch 2004; 449: 132-42.

[64] Tavares RG, Tasca CI, Santos CE, et al. Quinolinic acid stimulates synaptosomal glutamate release and inhibits glutamate uptake into astrocytes. Neurochem Int 2002; 40: 621-7.

[65] Tavares RG, Schmidt AP, Abud J, Tasca CI, Souza DO. In vivo quinolinic acid increases synaptosomal glutamate release in rats: reversal by guanosine. Neurochem Res 2005; 30: 439-44.

[66] Tavares RG, Schmidt AP, Tasca CI, Souza DO. Quinolinic acidinduced seizures stimulate glutamate uptake into synaptic vesicles from rat brain: effects prevented by guanine-based purines. Neurochem Res 2008; 33: 97-102.

[67] Torres FV, da Silva Filho M, Antunes C, et al. Electrophysiological effects of guanosine and MK-801 in a quinolinic acid-induced seizure model. Exp Neurol 2010; 221: 296-306.

[68] Franke H, Grummich B, Härtig W, et al. Changes in purinergic signaling after cerebral injury - involvement of glutamatergic mechanisms? Int J Devl Neuroscience 2006; 24: 123-32.

[69] Deutsch SI, Long KD, Rosse RB, Mastropaolo J, Eller J. Hypothesized deficiency of guanine-based purines may contribute to abnormalities of neurodevelopment, neuromodulation, and neurotransmission in Lesch-Nyhan syndrome. Clin Neuropharmacol 2005; $28: 28-37$.

[70] Di Iorio P, Ballerini P, Traversa $\mathrm{U}$, et al. The antiapoptotic effect of guanosine is mediated by the activation of the PI 3kinase/AKT/PKB pathway in cultured rat astrocytes. Glia 2004; 46: 356-68.

[71] Pettifer KM, Kleywegt S, Bau CJ, et al. Guanosine protects SHSY5Y cells against beta-amyloid-induced apoptosis. Neuroreport 2004; 15: 833-6.

[72] Oses JP, Cardoso CM, Germano RA, et al. Soluble NTPDase: An additional system of nucleotide hydrolysis in rat blood serum. Life Sci 2004; 74: 3275-84.

[73] Silva RG, Santos DS, Basso LA, et al. Purine nucleoside phosphorylase activity in rat cerebrospinal fluid. Neurochem Res 2004; 29: $1831-5$.

[74] Oses JP, Leke R, Portela LV, et al. Biochemical brain markers and purinergic parameters in rat CSF after seizure induced by pentylenetetrazol. Brain Res Bull 2004; 64: 237-42.

[75] Chen HS, Lipton SA. The chemical biology of clinically tolerated NMDA receptor antagonists. J Neurochem 2006; 97: 1611-26.

[76] Rubin MA, Jurach A, Costa EM, et al. GMP reverses the facilitatory effect of glutamate on inhibitory avoidance task in rats. Neuroreport 1996; 7: 2078-80.

[77] Roesler R, Vianna MR, Lara DR, Izquierdo I, Schmidt AP, Souza DO. Guanosine impairs inhibitory avoidance performance in rats. Neuroreport 2000; 11: 2537-40.

[78] Vinade ER, Izquierdo I, Lara DR, Schmidt AP, Souza DO. Oral administration of guanosine impairs inhibitory avoidance performance in rats and mice. Neurobiol Learn Mem 2004; 81: 137-43. 
[79] Saute JA, da Silveira LE, Soares FA, Martini LH, Souza DO, Ganzella M. Amnesic effect of GMP depends on its conversion to guanosine. Neurobiol Learn Mem 2006; 85: 206-12.

[80] Tort AB, Mantese CE, dos Anjos GM, et al. Guanosine selectively inhibits locomotor stimulation induced by the NMDA antagonist dizocilpine. Behav Brain Res 2004; 154: 417-22.

[81] Adams BW, Moghaddam B. Effect of clozapine, haloperidol, or M100907 on phencyclidine-activated glutamate efflux in the prefrontal cortex. Biol Psychiatry 2001; 50: 750-7.

[82] Moghaddam B, Adams B, Verma A, Daly D. Activation of glutamatergic neurotransmission by ketamine: a novel step in the pathway from NMDA receptor blockade to dopaminergic and cognitive disruptions associated with the prefrontal cortex. J Neurosci 1997; 17: 2921-7.

[83] Schmidt AP, Tort AB, Silveira PP, et al. The NMDA antagonist MK-801 induces hyperalgesia and increases CSF excitatory amino acids in rats: reversal by guanosine. Pharmacol Biochem Behav 2009; 91: 549-53.

[84] Schmidt AP, Tort AB, Souza DO, Lara DR. Guanosine and its modulatory effects on the glutamatergic system. Eur Neuropsychopharmacol 2008; 18: 620-2.

[85] Schmidt AP, Böhmer AE, Antunes C, et al. Anti-nociceptive properties of the xanthine oxidase inhibitor allopurinol in mice: role of A1 adenosine receptors. Br J Pharmacol 2009; 156: 163-72.

[86] Gudermann T, Schorneberg T, Schultz G. Functional and structural complexity of signal transduction via G-protein-coupled receptors. Ann Rev Neurosci 1997; 20: 399-427.

[87] Sharif NA, Roberts PJ. Regulation of cerebellar L-[3H]glutamate binding: influence of guanine nucleotides and $\mathrm{Na}+$ ions. Biochem Pharmacol 1981; 30: 3019-22.

[88] Butcher SS, Roberts PJ, Collins JF. Purine nucleotides inhibit the binding of DL- $[3 \mathrm{H}] \quad$ 2-amino-4-phosphonobutyrate (DL$[3 \mathrm{H}] \mathrm{APB})$ to L-glutamate-sensitive sites on rat brain membranes. Biochem Pharmacol 1986; 35: 991-4.

[89] Monahan JB, Hood WF, Michel J, Compton RP. Effects of guanine nucleotides on N-methyl-D-aspartate receptor-ligand interactions. Mol Pharmacol 1988; 34: 111-6.

[90] Hood WF, Thomas JW, Compton RP, Monahan JB. Guanine nucleotide modulation of $[3 \mathrm{H}] \mathrm{TCP}$ binding to the NMDA receptor complex. Eur J Pharmacol 1990; 188: 43-9.

[91] Rubin MA, Medeiros AC, Rocha PC, Livi CB, Ramirez G, Souza DO. Effect of guanine nucleotides on $[3 \mathrm{H}]$ glutamate binding and on adenylate cyclase activity in rat brain membranes. Neurochem Res 1997; 22: 181-7.

[92] Burgos JS, Barat A, Ramírez G. $\mathrm{Cl}^{-}$-dependent excitotoxicity is associated with ${ }^{3} \mathrm{H}_{2} \mathrm{O}$ influx in chick embryonic retina. Neuroreport 2000; 11: 3779-82.

[93] Burgos JS, Barat A, Ramírez G. $\mathrm{Ca}^{2+}$-dependent kainate excitotoxicity in the chick embryonic neural retina ex vivo. Neuroreport 2000; 11: 3855-8.

[94] Burgos JS, Aleu J, Barat A, Solsona C, Marsal J, Ramírez G. Kainate-triggered currents in Xenopus oocytes injected with chick retinal membrane fragments: Effect of guanine nucleotides. Invest Ophthalmol Vis Sci 2003; 44: 3124-9.

[95] Regner A, Ramírez G, Belló-Klein A, Souza DO. Effects of guanine nucleotides on glutamate-induced chemiluminescence in rat hippocampal slices submitted to hypoxia. Neurochem Res 1998; 23: 519-24.

[96] Tasca CI, Wofchuk ST, Souza DO, Ramirez G, Rodnight R. Guanine nucleotides inhibit the stimulation of GFAP phosphorylation by glutamate. Neuroreport $1995 ; 6: 249-52$.

[97] Tasca CI, Cardoso LF, Martini LH, Ramirez G, Souza DO. Guanine nucleotides inhibit cAMP accumulation induced by metabotropic glutamate receptor activation. Neurochem Res 1998; 23 : 183-8.

[98] Tasca CI, Cardoso LF, Souza DO. Effects of guanine nucleotides on adenosine and glutamate modulation of cAMP levels in optic tectum slices from chicks. Neurochem Int 1999; 34: 213-20.

[99] Tasca CI, Souza DO. Interaction of adenosine and guanine derivatives in the rat hippocampus: effects on cyclic AMP levels and on the binding of adenosine analogues and GMP. Neurochem Res 2000; 25: 181-8.

[100] Molz S, Tharine DC, Decker H, Tasca CI. GMP prevents excitotoxicity mediated by NMDA receptor activation but not by reversal activity of glutamate transporters in rat hippocampal slices. Brain Res 2008; 1231: 113-20.
[101] Aleu J, Barat A, Burgos J, Solsona C, Marsal J, Ramirez G. Guanine nucleotides, including GMP, antagonize kainate responses in Xenopus oocytes injected with chick cerebellar membranes. J Neurochem 1999; 72: 2170-6.

[102] Frizzo ME, Soares FA, Dall'Onder LP, Lara DR, Swanson RA, Souza DO. Extracellular conversion of guanine-based purines to guanosine specifically enhances astrocyte glutamate uptake. Brain Res 2003; 972: 84-9.

[103] Mendieta J, Ramirez G, Gago F. Molecular dynamics simulations of the conformational changes of the glutamate receptor ligandbinding core in the presence of glutamate and kainate. Proteins 2001; 44: 460-9.

[104] Mendieta J, Gago F, Ramirez G. Binding of 5'-GMP to the GluR2 AMPA receptor: insight from targeted molecular dynamics simulations. Biochemistry 2005; 44: 14470-6.

[105] Ciccarelli R, Di Iorio P, D'Alimonte I, et al. Cultured astrocyte proliferation induced by extracellular guanosine involves endogenous adenosine and is raised by the co-presence of microglia. Glia 2000; 29: 202-11.

[106] Gysbers JW, Rathbone MP. GTP and guanosine synergistically enhance NGF-induced neurite outgrowth from PC12 cells. Int J Devl Neuroscience 1996; 14: 19-34.

[107] Frizzo ME, Lara DR, Dahm KC, Prokopiuk AS, Swanson R, Souza DO. Activation of glutamate uptake by guanosine in primary astrocyte cultures. Neuroreport 2001; 12: 879-81.

[108] Traversa U, Bombi G, Di Iorio P, Ciccarelli R, Werstiuk ES, Rathbone MP. Specific $\left[{ }^{3} \mathrm{H}\right]$-guanosine binding sites in rat brain membranes. Br J Pharmacol 2002; 135: 969-76.

[109] Traversa U, Bombi G, Camaioni E, et al. Rat brain guanosine binding site. Biological studies and pseudo-receptor construction. Bioorg Med Chem 2003; 11: 5417-25.

[110] Frizzo ME, Schwalm FD, Frizzo JK, Soares FA, Souza DO. Guanosine enhances glutamate transport capacity in brain cortical slices. Cell Mol Neurobiol 2005; 25: 913-21.

[111] Dobolyi A, Reichart A, Szikra T, Nyitrai G, Kekesi KA, Juhasz G. Sustained depolarization induces changes in the extracellular concentrations of purine and pyrimidine nucleosides in the rat thalamus. Neurochem Int 2000; 37: 71-9.

[112] Gottfried C, Tramontina F, Goncalves D, et al. Glutamate uptake in cultured astrocytes depends on age: a study about the effect of guanosine and the sensitivity to oxidative stress induced by $\mathrm{H}_{2} \mathrm{O}_{2}$. Mech Ageing Dev 2002; 123: 1333-40.

[113] Thomazi AP, Godinho GF, Rodrigues JM, et al. Ontogenetic profile of glutamate uptake in brain structures slices from rats: sensitivity to guanosine. Mech Ageing Dev 2004; 125: 475-81.

[114] Moretto MB, Arteni NS, Lavinsky D, et al. Hypoxic-ischemic insult decreases glutamate uptake by hippocampal slices from neonatal rats: prevention by guanosine. Exp Neurol 2005; 195: 400-6.

[115] Tavares RG, Tasca CI, Santos CE, Wajner M, Souza DO, DutraFilho CS. Quinolinic acid inhibits glutamate uptake into synaptic vesicles from rat brain. Neuroreport 2000; 27: 249-53.

[116] Kim JK, Rathbone MP, Middlemiss PJ, Hughes DW, Smith RW Purinergic stimulation of astroblast proliferation: guanosine and its nucleotides stimulate cell division in chick astroblasts. J Neurosci Res 1991; 28: 442-55.

[117] Rathbone MP, Christjanson L, Deforge S, et al. Extracellular purine nucleosides stimulate cell division and morphogenesis: pathological and physiological implications. Med Hypotheses 1992; 37: 232-40.

[118] Rathbone MP, Middlemiss PJ, Gysbers JW, DeForge S, Costello P, Del Maestro RF. Purine nucleosides and nucleotides stimulate proliferation of a wide range of cell types. In vitro Cell Dev Biol 1992; 28A: 529-36.

[119] Gerrikagoitia I, Martínez-Millán L. Guanosine-induced synaptogenesis in the adult brain in vivo. Anat Rec (Hoboken) 2009; 292: 1968-75.

[120] Gysbers JW, Rathbone MP. Guanosine enhances NGF-stimulated neurite outgrowth in PC12 cells. Neuroreport 1992; 3: 997-1000.

[121] Rathbone MP, Middlemiss PJ, Andrew C, et al. The trophic effects of purines and purinergic signaling in pathologic reactions of astrocytes. Alzheimer Dis Assoc Disord 1998; 12: S36-45.

[122] Uemura Y, Miller JM, Matson WR, Beal MF. Neurochemical analysis of focal ischemia in rats. Stroke 1991; 22: 1548-53.

[123] Litsky ML, Hohl CM, Lucas JH, Jurkowitz MS. Inosine and guanosine preserve neuronal and glial viability in mouse spinal 
cord cultures during chemical hypoxia. Brain Res 1999; 821: 42632.

[124] Jurkowitz MS, Litsky ML, Browning Ml, Hohl CM. Adenosine, inosine, and guanosine protect glial cells during glucose deprivation and mitochondrial inhibition: correlation between protection and ATP preservation. J Neurochem 1998; 71: 535-48.

[125] Lara DR, Belmonte-de-Abreu P, Souza DO. Allopurinol for refractory aggression and self-inflicted behaviour. J Psychopharmacol 2000; 14: 81-3.

[126] Lara DR, Cruz MR, Xavier F, Souza DO, Moriguchi EH. Allopurinol for the treatment of aggressive behaviour in patients with dementia. Int Clin Psychopharmacol 2003; 18: 53-5.

[127] Brunstein MG, Ghisolfi ES, Ramos FL, Lara DR. A clinical trial of adjuvant allopurinol therapy for moderately refractory schizophrenia. J Clin Psychiatry 2005; 66: 213-9.

[128] Machado-Vieira R, Lara DR, Souza DO, Kapczinski F. Therapeutic efficacy of allopurinol in mania associated with hyperuricemia. $\mathbf{J}$ Clin Psychopharmacol 2001; 21: 621-2.

[129] Machado-Vieira R, Soares JC, Lara DR, et al. A double-blind, randomized, placebo-controlled 4-week study on the efficacy and safety of the purinergic agents allopurinol and dipyridamole adjunctive to lithium in acute bipolar mania. J Clin Psychiatry 2008; 69: $1237-45$.
[130] Akhondzadeh S, Safarcherati A, Amini H. Beneficial antipsychotic effects of allopurinol as add-on therapy for schizophrenia: a double blind, randomized and placebo controlled trial. Prog Neuropsychopharmacol Biol Psychiatry 2005; 29: 253-9.

[131] Akhondzadeh S, Milajerdi MR, Amini H, Tehrani-Doost M. Allopurinol as an adjunct to lithium and haloperidol for treatment of patients with acute mania: a double-blind, randomized, placebocontrolled trial. Bipolar Disord 2006; 8: 485-9.

[132] Zagnoni PG, Bianchi A, Zolo P, et al. Allopurinol as add-on therapy in refractory epilepsy: a double-blind placebo-controlled randomized study. Epilepsia 1994; 35: 107-12.

[133] Togha M, Akhondzadeh S, Motamedi M, Ahmadi B, Razeghi S. Allopurinol as adjunctive therapy in intractable epilepsy: a doubleblind and placebo-controlled trial. Arch Med Res 2007; 38: 313-6.

[134] Jiang S, Bendjelloul F, Ballerini P, et al. Guanosine reduces apoptosis and inflammation associated with restoration of function in rats with acute spinal cord injury. Purinergic Signal 2007; 3:41121.

[135] Jiang S, Khan MI, Lu Y, et al. Guanosine promotes myelination and functional recovery in chronic spinal injury. Neuroreport 2003; 14: 2463-7.

[136] Chang R, Algird A, Bau C, Rathbone MP, Jiang S. Neuroprotective effects of guanosine on stroke models in vitro and in vivo. Neurosci Lett 2008; 431: 101-5.

(C) Schmidt and Souza; Licensee Bentham Open.

This is an open access article licensed under the terms of the Creative Commons Attribution Non-Commercial License (http://creativecommons.org/licenses/by-nc/3.0/) which permits unrestricted, non-commercial use, distribution and reproduction in any medium, provided the work is properly cited. 